\title{
Case Report: A Rare Variety of Type 4 Monteggia Fracture Dislocation in a 28 Years Old Man
}

\author{
Ranadeb Bandyopadhyay ${ }^{1 *}$, Arindam Mukherjee ${ }^{2}$ \\ ${ }^{1}$ Department of Orthopaedics, Bankura Sammilani Medical College, Bankura, India; ${ }^{2}$ Bankura Sammilani Medical College, Bankura, \\ India. \\ Email: *ranadeb.b@rediffmail.com, arindamm02@gmail.com
}

Received March $6^{\text {th }}, 2012$; revised April 20 $0^{\text {th }}, 2012$; accepted May $8^{\text {th }}, 2012$

\begin{abstract}
A Monteggia fracture is a fracture of the proximal ulna coupled with a radial head dislocation. Numerous classification systems have been developed to characterize these fractures. Bado classification is most commonly used. Bado type 1 Monteggia fractures are most common while type $3 \& 4$ are rare entities. In our case, after confirming the diagnosis as type 4 Bado Monteggia fracture dislocation, patient was posted for open reduction and internal fixation. The ulnar and radial fractures were rigidly fixed with 3.5 compression plate and ulnar length restored. However, the radial head was still found to be dislocated anteriorly on fluoroscopy. An attempt of closed reduction of the radial head failed. Open reduction of the radial head was performed under image intensifier and fixed with a Kirschner's wire followed by immobilization in hyperflexion for 2 weeks. The patient followed up at the end of 2 weeks, 4 weeks and 6 weeks following injury and gradual mobilisation of the elbow joint was made. Further follow up at 6 months and 1 year showed complete return of routine function. As soon as the ulnar length is restored by rigid internal fixation, radial head is itself reduced in majority of Monteggia fracture dislocation where only ulnar fracture is involved. However, in cases of both bone forearm fracture with radial head dislocation, even after restoring the length of ulna and radius, operative reducetion of radial head is essential.
\end{abstract}

Keywords: Monteggia Fracture; Bado Classification; Rigid Fixation; Operative Reduction

\section{Introduction}

Fractures of the proximal ulna with a concomitant dislocation of the radial head are uncommon injuries that comprise $5 \%$ of all forearm fractures. The injury was first described by Monteggia in 1814. Typically a patient with these types of injuries will complain of pain around the elbow and mechanical block to elbow flexion and forearm rotation [1]. It is important to perform a careful examination of the nerves, especially the posterior interosseous nerve with a reported incidence as high as $17 \%$ [2].

The combination of injuries known as Monteggia fracture-dislocation is an often treacherous condition to treat. The Monteggia fracture and its variant can be treated conservatively in children, but routinely requires open reducetion in adults. The goal of treatment of a Monteggia injury is rigid fixation of fractured ulna which relocates the dislocated radial head [3]. Any residual angulation of the ulna fracture predisposes to subsequent redislocation or subluxation of the radial head. In the adult patient open reduction and internal fixation should be used.

${ }^{*}$ Corresponding author.

\section{Case Report}

A 28 years old male patient presented with severe pain and swelling of the right forearm following high velocity road traffic accident while driving his motorbike 1 day back. He also complained of developing deformity of his right forearm following the accident. No complaint of any neural deficit was present.

On examination, severe tenderness along with swelling was noted at the upper third of the right forearm. Bony crepitation and abnormal movement was palpable at the same site. Movement at the right elbow joint was restricted. No neurovascular deficit was noted. A provisional diagnosis of fracture both bone right forearm was made initially.

A radiograph of the right forearm in antero-posterior and lateral views showing both the wrist and elbow joint were obtained immediately. Radiograph showed fracture of proximal third of ulna, anterior dislocation of the radial head and fracture of proximal third of the radius of the right side. The patient was diagnosed to have an uncommon variety of Monteggia fracture-dislocation Bado type 4 (Figures 1 and 2).

The patient was immediately posted for open reduction 


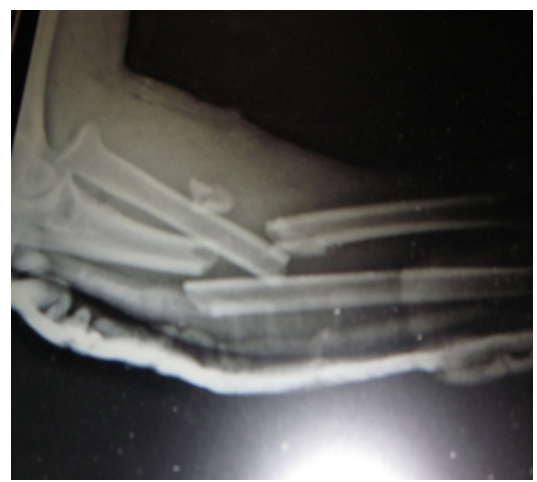

Figure 1. Pre-operative radiograph of the right forearm showing fractures of proximal third of the radius and ulna with anterior dislocation of the radial head.

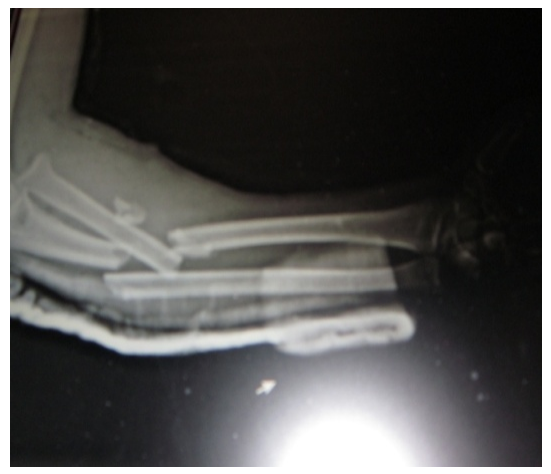

Figure 2. Pre-operative radiograph of the right forearm showing fractures of proximal third of the radius and ulna with anterior dislocation of the radial head along with normal anatomical wrist joint.

and internal fixation of the Monteggia fracture-dislocation under brachial block. The fractured ulna and radius were rigidly fixed with 3.5 small dynamic compression plate and 3.5 cortical screws. However, in spite of rigid fixation and restoration of ulnar length, the radial head was still found to be dislocated anteriorly on fluoroscopy (Figure 3). An attempt of closed reduction was made. However, stable reduction of the radial head could not be achieved. Open reduction of the dislocated radial head was then performed and fixed with a Kirschner's wire (Figure 4). Radial collateral ligament was repaired. Reduction was confirmed under image intensifier. The forearm was then immobilized in an above elbow dorsal splint in hyperflexion.One dose of parenteral antibiotics (inj. Cefuroxime and inj. Gentamycin) was given preoperatively followed by one dose intra-operatively and another dose post-operatively. This was followed by oral antibiotics of the same composition for another 5 days. The patient was followed up at the end of 2 weeks with radiographs. Wound healing was satisfactory and radiographs showed restored anatomy. Stitch off done, Kirschner's wire removed, dorsal splint discarded and collar and cuff sling applied (Figure 5). Gradual mobilization of supination and pronation movements started. Patient was again followed up at 4 weeks with radiograph. Gentle pronation and supination movements were permitted, but extension was not permitted beyond 90'. At 6 weeks, gradual extension movement beyond 90' was started. The patient was again followed up at the end of 6 months and 1 year with serial radiographs. Radiographs confirmed maintenance of satisfactory reduction and full range of motion returned at the right elbow joint (Figures 6-9).

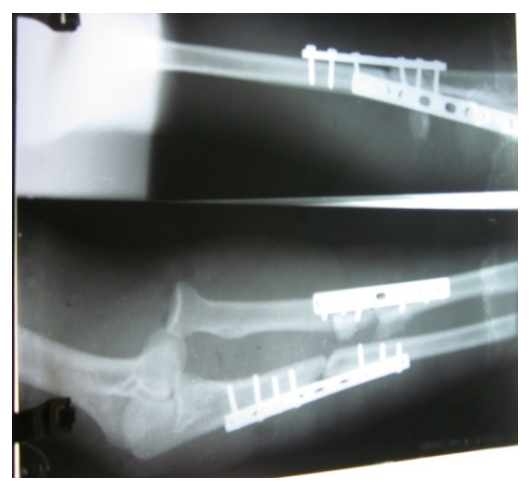

Figure 3. Initial per-operative radiograph showing plate osteosynthesis of both ulna and radius with persistent anterior dislocation of the radial head.

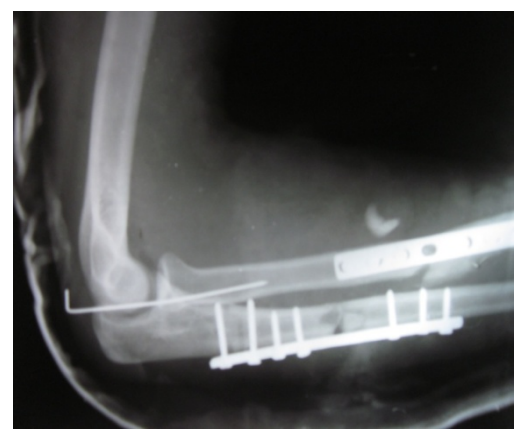

Figure 4. Final post-operative radiograph showing reduction of the dislocated radial head with a Kirschner's wire along with plate osteosynthesis of radius and ulna.

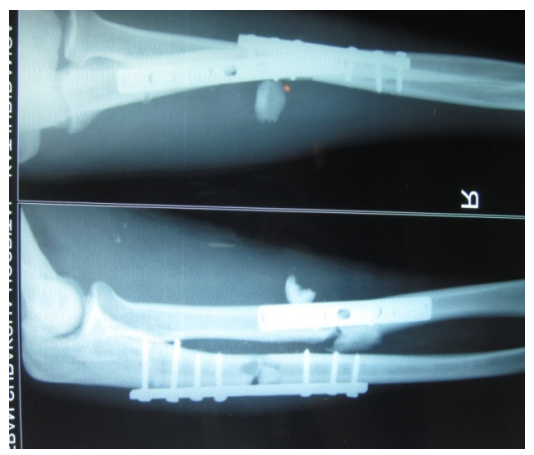

Figure 5. Radiograph of the right forearm after removal of Kirschner's wire at 2 weeks follow up with normal congruence of radio-capitellar joint and restoration of length of both radius and ulna. 


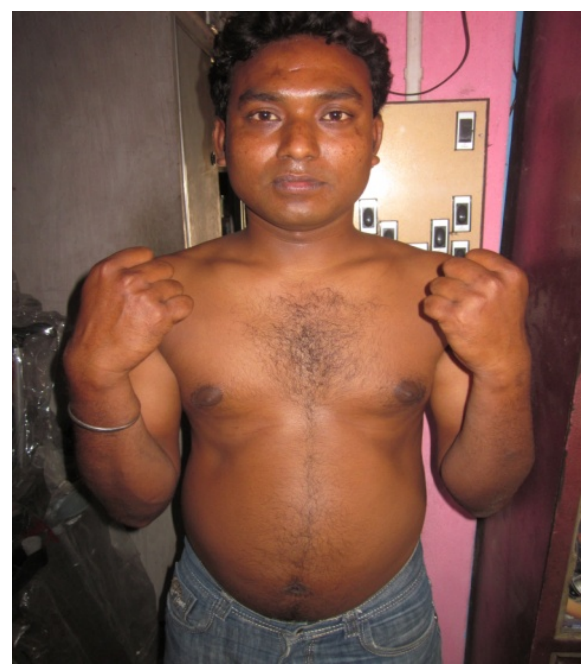

Figure 6. Clinical photograph at the end of 1 year showing full flexion.

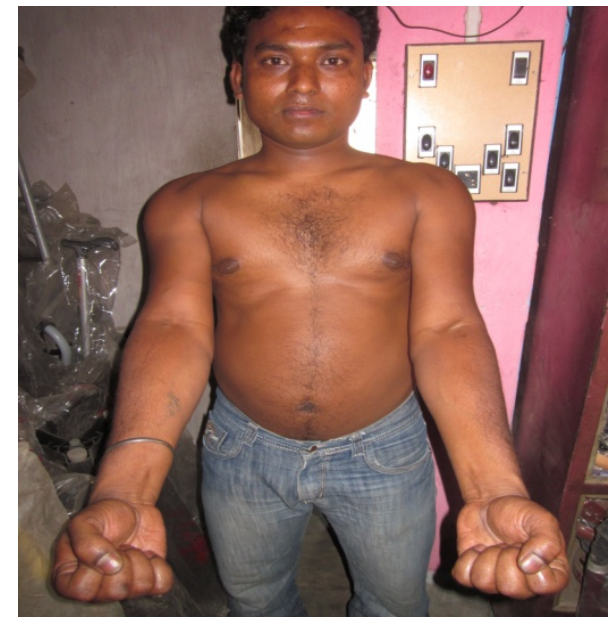

Figure 7. Clinical photograph at the end of 1 year showing full extension.

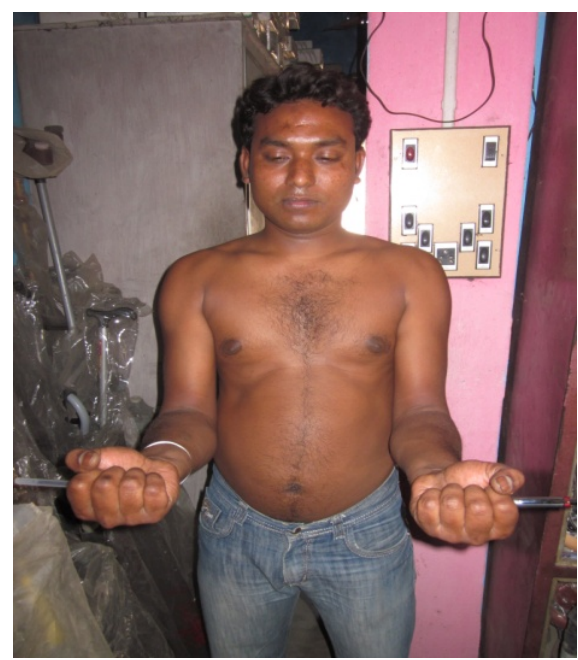

Figure 8. Clinical photograph at the end of 1 year showing full supination.

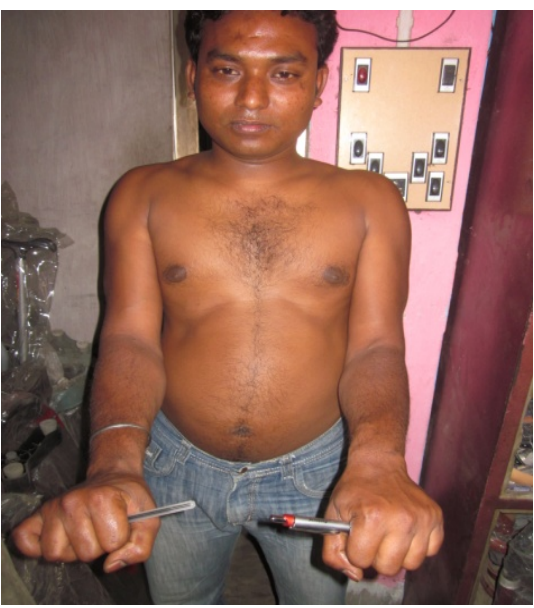

Figure 9. Clinical photograph at the end of 1 year showing full pronation.

\section{Discussion}

Monteggia fractures are rare but commonly discussed lesions, with increasing complications due to late diagnosis [4]. A Monteggia fracture is a fracture of the proximal ulna coupled with a radial head dislocation. These fractures are an uncommon class of forearm fractures. Numerous classification systems have been developed to characterize these fractures, with the Bado classification being the most common. Bado type 1 Monteggia fractures are most common while type $3 \& 4$ are uncommon phenomenon [5]. Equivalent Monteggia can occur by associated lesions such as olecranon fracture or radial neck or head fracture.Several mechanisms of injury probably exists, including direct blows to the ulnar aspect of the forearm and a fall with hyperpronation and hyperextension, with the strong supinating force of the biceps pulling the radial head anteriorly as the fracture of the ulna is produced by the compression forces of the fall [6].

Elbow radiographs are the primary diagnostic modality, demonstrating dislocation when a line drawn extending through the radial head from the radial shaft does not penetrate the capitellum in all views [7] Adolescents often achieve a better prognosis. Non-operative management with closed reduction and cast immobilization often prevails in pediatric patients, dictated by the pattern of the ulnar fracture more so than the direction of the radial head dislocation [8]. However, in adults, operative intervention is frequently indicated because angulation and shortening of the ulna often occur after closed reduction. Although the orthopedic community's understanding of these fractures has evolved, the fractures themselves remain a challenging clinical phenomenon [9].

The treatment plan usually varies depending on the situation. For acute injuries in which the dislocation of the radial head can be reduced by closed methods, open reduction of the dislocation is not indicated but the frac- 
ture of the ulna is rigidly fixed. In the proximal third of the ulna, where the medullary canal is large, a compression plate is used. In the middle third, where the medullary canal is small, either a compression plate or a triangular intra-medullary nail is used [10]. Careful intra-operative radiographic analysis of the radio-capitellar joint must be made after fixation of the ulnar shaft fracture. Subluxation of the radial head requires open reducetion [10]. For old injuries ( $\geq 6$ weeks old) in which the dislocation of the radial head has never been reduced or in which insufficient fixation of the fracture of the ulna has allowed angulation of the fracture and redislocation of the radial head, the radial head is excised. If the ulna is sufficiently angulated or ununited, it is rigidly fixed with compression plate and supplemented with bone grafts. Alternatively, if elbow joint instability is present with a coronoid fracture or collateral ligament damage, the radial head is reduced, and the ulna is repaired with the apex posteriorly angled plate [10].

In our case, after confirming the diagnosis as type 4 Bado Monteggia fracture dislocation, patient was posted for open reduction and internal fixation. The ulnar and radial fractures were rigidly fixed with 3.5 compression plate. The radial head was still found to be dislocated anteriorly on fluoroscopy. An attempt of closed reduction of the radial head was tried, but it failed. Open reduction of the radial head was performed under image intensifier and fixed with a Kirschner's wire. The forearm was then immobilized in an above elbow dorsal splint in hyperflexion. The patient followed up at the end of 2 weeks when the Kirschner's wire was removed and stitch off done followed by gradual mobilisation of the elbow joint. Further follow up at 6 months and 1 year showed complete return of routine function.

Complex elbow dislocation associated with radial and ulnar diaphyseal fractures is a rare combination. Monteggia type 4 fracture-dislocations are complex injuries with typical specific fracture fragments. Anatomic fixation of all injury components and avoidance of complications where possible can lead to a good outcome in these challenging injuries. The proximal third fractures of the ulna and radius needs rigid fixation usually with a compression plate. The dislocated radial head usually reduces following rigid fixation of the fracture and restoration of ulnar length. However, occasionally as in our case where both radius and ulna fracture present, it may fail to do so. In these situation, open reduction and internal fixation (with Kirschner's wire) of the dislocated radial head is required.

\section{REFERENCES}

[1] A. Stitgen, J. J. McCarthy, B. A. Nemeth, K. Garrels and K. J. Noonan, "Ulnar Fracture with Late Radial Head Dislocation: Delayed Monteggia Fracture," Orthopedics, Vol. 35, No. 3, 2012, pp. 434-437.

[2] B. G. Beutel, "Monteggia Fractures in Pediatric and Adult Populations," Orthopedics, Vol. 35, No. 2, 2012, pp. 138144. doi:10.3928/014774477-20120123-32

[3] I. Irubetagoyena, T. Lopez and A. Autefage, "Type IV Monteggia Fracture in a Cat," Veterinary and Comparative Orthopaedics and Traumatology, Vol. 24, No. 6, 2011, pp. 483-486. doi:10.3415/VCOT-11-01-0005

[4] A. P. Singh, I. K. Dhammi, A. K. Jain, R. Raman and P. Modi, "Monteggia Fracture Dislocation Equivalents: Analysis of Eighteen Cases Treated by Open Reduction and Internal Fixation," Chinese Journal of Traumatology, Vol. 14, No. 4, 2011, pp. 221-226.

[5] S. Ramesh and Y. J. Lim, "Complex Elbow Dislocation Associated with Radial and Ulnar Diaphyseal Fractures: A Rare Combination," Strategies in Trauma and Limb Reconstruction, Vol. 6, No. 2, 2011, pp. 97-101. doi:10.1007/s11751-011-0112-5

[6] D. M. Beingessner, S. E. Nork, J. Agel and D. Viskontas, "A Fragment-Specific Approach to Type IID Monteggia Elbow Fracture-Dislocations," Journal of Orthopaedic Trauma, Vol. 25, No. 7, 2011, pp. 414-419. doi:10.1097/BOT.0b013e3181fc6255

[7] D. Ristić, N. Jovanović, V. Cvetković, B. Stanković and B. Vracević, "Bilateral Monteggia Fracture in Adults," Vojnosanitetski Pregled, Vol. 68, No. 4, 2011, pp. 363365. doi:10.2298/VSP1104363R

[8] Z. Vukasinović, V. Jovanović, D. Mitović and N. Slavković, "Treatment of Missed Monteggia Lesion in Children: Case Report," Srpski Arhiv Za Celokupno Lekarstvo, Vol. 139, No. 1-2, 2011, pp. 99-102. doi:10.2298/SARH1102099V

[9] W. Min, M. Cohn and T. M. McLaurin, "Incarceration of the Radial Head Associated with a Radial Head Fracture, Radio Capitellar Dislocation, and Proximal Radioulnar Translocation," Journal of Orthopaedic Trauma, Vol. 25, No. 5, 2011, pp. e43-e46. doi:10.1097/BOT.0b013e3181e574da

[10] A. H. Crenshaw Jr. and E. A. Perez, "Campbell's Operative Orthopaedics: Chapter 54-Fractures of the Shoulder, Arm and Forearm," 11th Edition, Mosby Elsevier, Missouri, 2008, pp. 3420-3424. 